\title{
Autophagy in insulin-induced hypoglycaemic neuropathy
}

\author{
Simin Mohseni
}

\section{Linköping University Post Print}

N.B.: When citing this work, cite the original article.

Original Publication:

Simin Mohseni, Autophagy in insulin-induced hypoglycaemic neuropathy, 2011, PATHOLOGY, (43), 3, 254-260.

http://dx.doi.org/10.1097/PAT.0b013e328343c992

Copyright: Informa Healthcare / Lippincott, Williams \& Wilkins http://www.lww.com/

Postprint available at: Linköping University Electronic Press

http://urn.kb.se/resolve?urn=urn:nbn:se:liu:diva-67707 


\section{Autophagy in insulin-induced hypoglycaemic neuropathy}

Simin Mohseni

Division of Cell Biology, Department of Clinical and Experimental Medicine, Faculty of Health Sciences, Linköping University, Linköping, Sweden

\section{Running head: Autophagy in hypoglycaemic neuropathy}

Correspondence: $\quad$ Simin Mohseni

Division of Cell Biology

Faculty of Health Sciences

Linköping University

SE-581 85 Linköping, Sweden

E-mail: $\quad$ simin.mohseni@liu.se

Telephone: $\quad+46101034144$

Fax: $\quad$ +46 101033192 


\section{Abstract}

Aim: Autophagy in neurons has been linked to a growing number of pathological conditions in the CNS, but the role of this process in peripheral neuropathy has received little attention. This study aimed to determine whether autophagy is involved in development of peripheral neuropathy in hypoglycaemic diabetic rats.

Methods: The lateral plantar nerves, ventral roots, and dorsal roots of insulin-treated diabetic hypoglycaemic rats were examined for structural signs of autophagy by electron microscopy.

Results: Autophagy-associated vacuoles were found in myelinated axons exhibiting early pathological changes but not in the associated Schwann cells. When the damaged axons degenerated, their associated Schwann cells gradually died and were cleared from the endoneurium by macrophages. During axonal regeneration, extensive signs of autophagy-related structures such as autophagophores appeared in regenerating axons and in the cytoplasm of the associated Schwann cells in the Band of Büngner.

Conclusion: Autophagy occurs in hypoglycaemic peripheral nerves in association with axonal de- and regeneration. The extensive signs of autophagy in regenerated axons suggest that autophagy may play a role in survival of the new axons.

Keywords: Autophagy, diabetes, electron microscopy, hypoglycaemia, peripheral neuropathy. 


\section{INTRODUCTION}

In 1850, Augustus Waller described the morphological consequence of cutting the peripheral nerves of frogs, which is known as Wallerian degeneration. ${ }^{1}$ Briefly, after such traumatic injury, the distal parts of the axons and their associated myelin degenerate. Thereafter, macrophages invade the endoneurium and remove axonal and myelin debris. Also, Schwann cells proliferate and become organised in columns within the basal lamina of the axons they had surrounded, and these structures are called bands of Büngner (or Schwann tubes). In this way, the Schwann cells guide regenerating axons to the distal nerve stumps. Axonal degeneration can also be caused by metabolic, toxic, or ischemic events, and if the continuity of a nerve fibre is interrupted by these events, the process is called Wallerian-like degeneration. It is presently assumed that axonal degeneration is an active self-destruction event rather than a passive clearance of damaged nerve fibres. Biochemical changes that are consistent with apoptosis, such as caspase activation, are involved in cortical synapses in response to staurosporine which induces apoptosis by activating caspase- $3 .{ }^{2}$ However, Finn and colleagues ${ }^{3}$ have reported that degeneration in the peripheral nerves does not include caspase activation. Thus it is plausible that apoptosis is involved in degeneration of the Schwann cells that have lost their axonal connections,${ }^{4-6}$ and macrophages may play an active role in removal of myelin by opsonisation..$^{7-9}$ Despite the significance, the molecular mechanism of axonal degeneration is not well understood.

Autophagy (refers to macroautophagy) occurs when a cell handles its own damaged organelles and proteins by enclosing them within vesicles and delivering them to the 
Iysosomal compartment for degradation and reuse. More precisely, doublemembrane vesicles that are synthesised in the cytoplasm engulf the damaged intracellular components, and the vacuoles that are formed in that way are termed autophagosomes. The presence of these vesicles internalising intracellular organelles such as mitochondria has been regarded as an ultrastructural sign of autophagosomes. These vacuoles subsequently fuse with lysosomes (i.e., autophagolysosomes) in a process which leads to digestion of their contents by lysosomal enzymes. ${ }^{10}$ Lysosomes are cytoplasmic vacuoles that have a low internal $\mathrm{pH}$ of 4.8, and they contain enzymes that are involved in digestion of intracellular and phagocytosed extracellular components to yield simple compounds. In the electron microscope, lysosomes are seen as electron-dense single-membrane vacuoles enclosing fragments of structures such as membranes or mitochondria.

The autophagy pathway is believed to play an important role in maintenance of cell function under conditions of stress (e.g., nutrient deprivation). Notably, autophagy has been linked to various pathological conditions in humans. For example, accumulation of autophagosomes in neurons of the brain has been observed in a number of neurodegenerative disorders, including Alzheimer's ${ }^{11}$ and Parkinson's ${ }^{12}$ diseases. It has been suggested that autophagy provides protection against neurodegeneration by preventing neurons from undergoing apoptosis. ${ }^{3,14}$ However, there is also cellular evidence indicating that autophagy promotes neuronal cell death. ${ }^{15,16}$ Hopefully the uncertain role of autophagy in the destiny of stressed neurons will be clarified in the near future.

Research on the role of autophagy in the nervous system has been focused chiefly on neurons in the CNS and diseases that affect the brain, and little attention has been given to autophagy in peripheral nerve injuries. Yang and colleagues ${ }^{17}$ found 
that autophagy was induced in sympathetic neurites after axonal degeneration following NGF deprivation in vitro. In vivo, autophagy in axons was observed during regeneration of transected rat sciatic nerves. ${ }^{18}$ We have previously observed that hypoglycaemia caused Wallerian-like degeneration of large myelinated axons in the peripheral nerves of diabetic rats given intensive insulin treatment. ${ }^{19-23}$ Despite this extensive axonal breakdown, the number of axons in these nerves did not decrease dramatically, because many axons were regenerated. This indicates that neurons in the peripheral nerves survived the insult and were able to sprout new axonal branches, which suggests that hypoglycaemic neuropathy consists primarily of distal axonopathy rather than neuronopathy. The present study addressed the question of whether autophagy may have played a role in the axonal degeneration and/or regeneration that was observed in the peripheral nerves in our previous investigations. Apparently no earlier studies have examined autophagy in association with hypoglycaemic neuropathy in diabetes. In the current work, electron microscopy was used to search for morphological signs of autophagy in hypoglycaemic neuropathy, which revealed autophagy-related structures in degenerating axons but not in their associated Schwann cells. However, during regeneration, signs of autophagy were observed in both regenerating axons and their Schwann cells. 


\section{MATERIALS AND METHODS}

The experimental protocol was approved by the local Ethics Committee for Animal Experimentation on 21 February 2008 (protocol number: Dnr 20-08). The postal address of this committee is as follows: Linköpings djurförsöksetiska nämnd, Linköpings tingsrätt, P.O. box 365, SE-58103 Linköping, Sweden.

Female BB/Wor rats were maintained in cages with soft bedding and free access to rat chow and water. Almost $70 \%$ of animals of this strain develop diabetes spontaneously after autoimmune inflammation in the pancreas, and hence these rats represent one of the best animal models of Type 1 diabetes. ${ }^{24}$ After arrival at the laboratory, the rats were weighed and their blood glucose was measured twice weekly before the onset of the disease, which was signalled by weight loss and high blood glucose levels. The diabetic rats were treated with appropriate amounts of insulin via subcutaneous implants (Linplant $\Theta$, LinshinCanada, Ontario, Canada) to achieve a subnormal glucose level during the treatment period (16-20 weeks). Each such implant releases 2 units of insulin per day for almost 4 weeks. Details of the insulin implants and the implantation procedure have been reported elsewhere. ${ }^{23}$ After onset of the disease, blood glucose was measured 2-3 times a week in hypoglycaemic rats and once a week in controls by using a Glucometer Elite $\AA_{\text {device }}$ (Bayer, Leverkusen, Germany).

At the end of the experiment, the animals were sacrificed by deep anaesthesia and perfused with Tyrode's solution followed by $5 \%$ glutaraldehyde (Taab, Berks, England) in phosphate buffer. Samples of the lateral plantar nerves (LPNs) at the ankle level, dorsal roots (DRs), and ventral roots (VRs) were taken from age-matched 
healthy rats $(n=5)$ and hypoglycaemic animals (LPNs, $n=6$; DRs, $n=4$; VRs, $n=$ 4), and then prepared for electron microscopic examination. Briefly, the samples were postfixed, osmicated (2\%), dehydrated in acetone, embedded in Vestopal polyester, and cut in semi-thin sections (approximately $0.5 \mu \mathrm{m}$ thick). The sections were contrasted with uranyl acetate and lead citrate, and examined in a JOEL JEM 1200 EX electron microscope. The number of degenerated nerve fibres was determined indirectly by counting the fragments of myelin debris. Regeneration units are formed when axons in the proximal part of an injury sprout new branches called regenerated axons. Here, the number of such units containing small axons that were both myelinated and unmyelinated and enclosed by a basal lamina was counted in the LPN, DR, and VR in one semi-thin section from each animal. Attention was paid to examining the occurrence of autophagy-related structures in the nerves. All evaluations were performed on coded samples. The weight and blood glucose levels of the animals are expressed as mean \pm SD. 


\section{RESULTS}

\section{Metabolic Status}

At the time of arrival at the laboratory, the rats were 35-65 days old, weighed on average $126.5 \pm 16.3 \mathrm{~g}$, and had a normal blood glucose level $(5.3 \pm 0.3 \mathrm{mmol} / \mathrm{l})$. A high blood glucose value $(21.9 \pm 4.9 \mathrm{mmol} / \mathrm{l})$ at an average age of $78 \pm 4$ days indicated the onset of diabetes in affected rats. In controls, blood glucose was tightly regulated and had a median value of $5.5 \pm 0.5 \mathrm{mmol} / \mathrm{l}$. In hypoglycaemic animals, blood glucose during the period of insulin therapy varied between 1.5 and $23 \mathrm{mmol} / \mathrm{l}$, with a median value of $4.1 \pm 3.3$; the highest levels represent a short period of time before an exhausted implant was replaced with a new one (Fig. 1). At termination of the experiment, the healthy animals and the diabetic hypoglycaemic rats had average weights of $243.6 \pm 4.5 \mathrm{~g}$ and $224.1 \pm 7.4 \mathrm{~g}$, respectively. All rats showed normal behaviour during the experimental period.

\section{Findings in nerves from healthy rats}

Examination of LPN (Fig. 2a), DR, and VR sections confirmed normal morphology of these tissues, as described previously. ${ }^{21,23}$ Briefly, the LPNs and DRs contained large, medium-sized, and small myelinated as well as unmyelinated axons. The picture in the VRs was dominated by large myelinated axons, and some mediumsized and a few unmyelinated axons were also observed. In myelinated axons of these nerves, single-membrane vesicles that had diameters of approximately $0.2-0.7$ $\mu \mathrm{m}$ and contained axoplasmic inclusions or had an electron lucid appearance were seen in a few axons. 


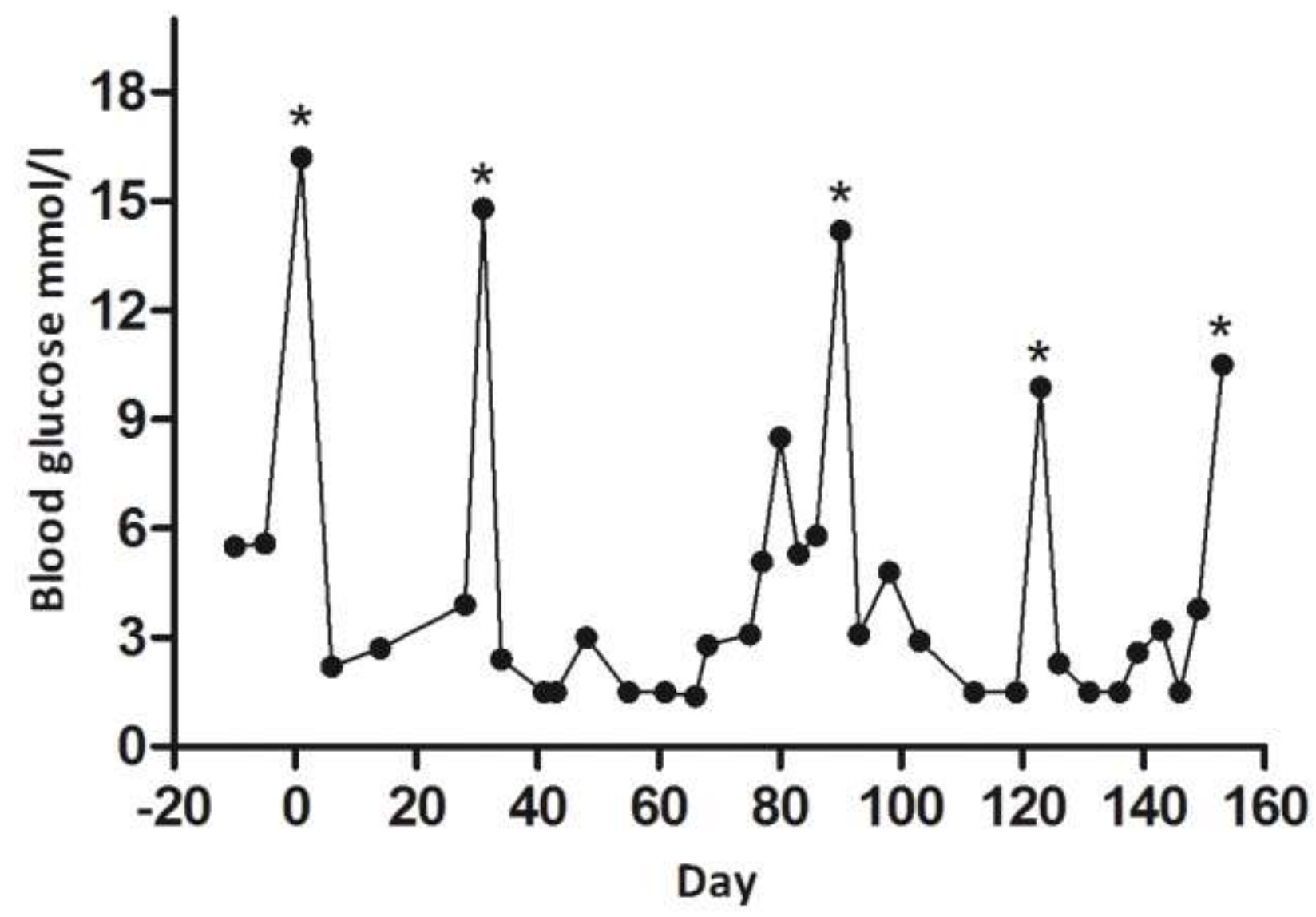

Fig. 1 Blood glucose levels in a representative insulin-treated hypoglycaemic diabetic rat treated with insulin implants for 5 months. Day 0 indicates the onset of diabetes and asterisks indicate when the rat received a new insulin implant.
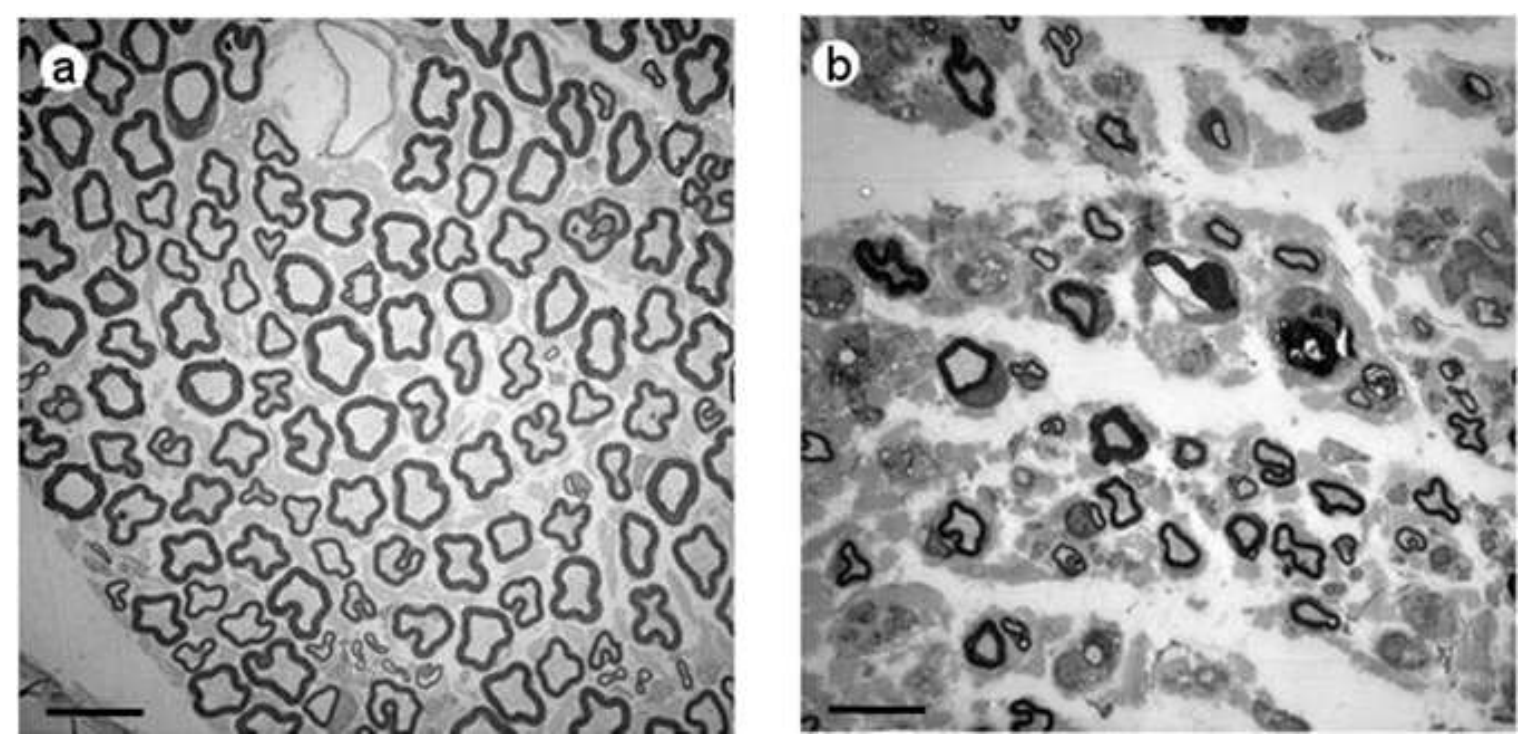

Fig. 2a-b Electron micrographs of transverse sections of the lateral plantar nerve (LPN) from a healthy rat (a), showing the normal appearance of myelinated fibres, and from an insulin-treated diabetic hypoglycaemic rat (b). Note the scarcity of large myelinated fibres and occurrence of myelin debris (arrows). Scale bars $=1 \mu \mathrm{m}$. 


\section{Findings in nerves from hypoglycaemic rats}

Extensive signs of de- and regeneration of large myelinated axons were observed in the LPNs (Fig. 2b) and VRs, whereas the DRs showed only a few degenerated fibres (Table 1). In the LPN and VR sections, the number of large myelinated axons was reduced, and the nerve fibres displayed varying degrees of pathology. The early stage of nerve fibre degeneration was manifested by axonal shrinkage and myelin sheath segregation, whereas the absence of axons and occurrence of collapsed myelin sheaths constituted the hallmarks of a later stage of degeneration.

Table 1 The number of cells involved in degradation of myelin and the number of regeneration units counted in one semi-thin section from each animal and nerve

\begin{tabular}{|l|l|l|l|l|}
\hline No. of rats & Nerve & $\begin{array}{l}\text { Schwann } \\
\text { cells }\end{array}$ & Macrophages & $\begin{array}{l}\text { Regeneration } \\
\text { units }\end{array}$ \\
\hline 6 & LPN & 11 & 7 & 47 \\
\hline 4 & VR & 21 & 29 & 2 \\
\hline 4 & DR & 8 & 3 & 0 \\
\hline
\end{tabular}

LPN = lateral plantar nerve; $\mathrm{VR}=$ ventral root; $\mathrm{DR}=$ dorsal root

Notice the relative few Schwann cells and macrophages involved in axonal degeneration and a high number of regeneration units in the hypoglycaemic LPNs. In the VRs, there are many Schwann cells and macrophages, but few regeneration units. Together, this data suggests that axonal degeneration starts at distal level and develops to the proximal part of the nerves

Early in axonal degeneration, the axolemma was partly detached from myelin sheaths, invaded the axoplasm, and formed vesicle-like structures (Fig. 3a). The axoplasm also contained lucid vesicles of different sizes, many small (diam. $\leq 0.2$ $\mu \mathrm{m}$; not shown) and some larger (diam. $\geq 0.5 \mu \mathrm{m}$; Fig. 3b). At this initial stage of degeneration, the axoplasm of large myelinated axons showed a few autophagyrelated structures, as well as enlarged mitochondria with disorganised and partly disrupted cristae (Fig. 3c-d), and these damaged organelles were often seen in the 
vicinity of autophagic vacuoles. Schwann cells had normal morphology except for the occurrence of single-membrane vesicles in the cytoplasm.
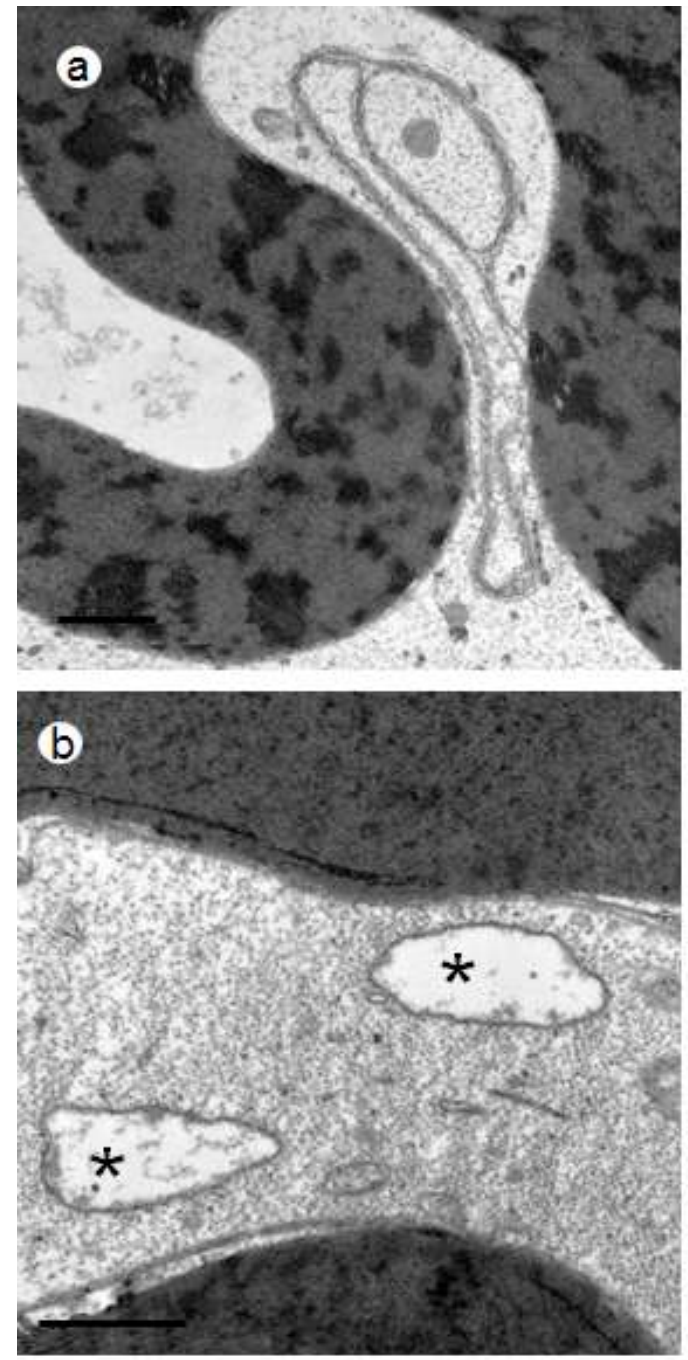
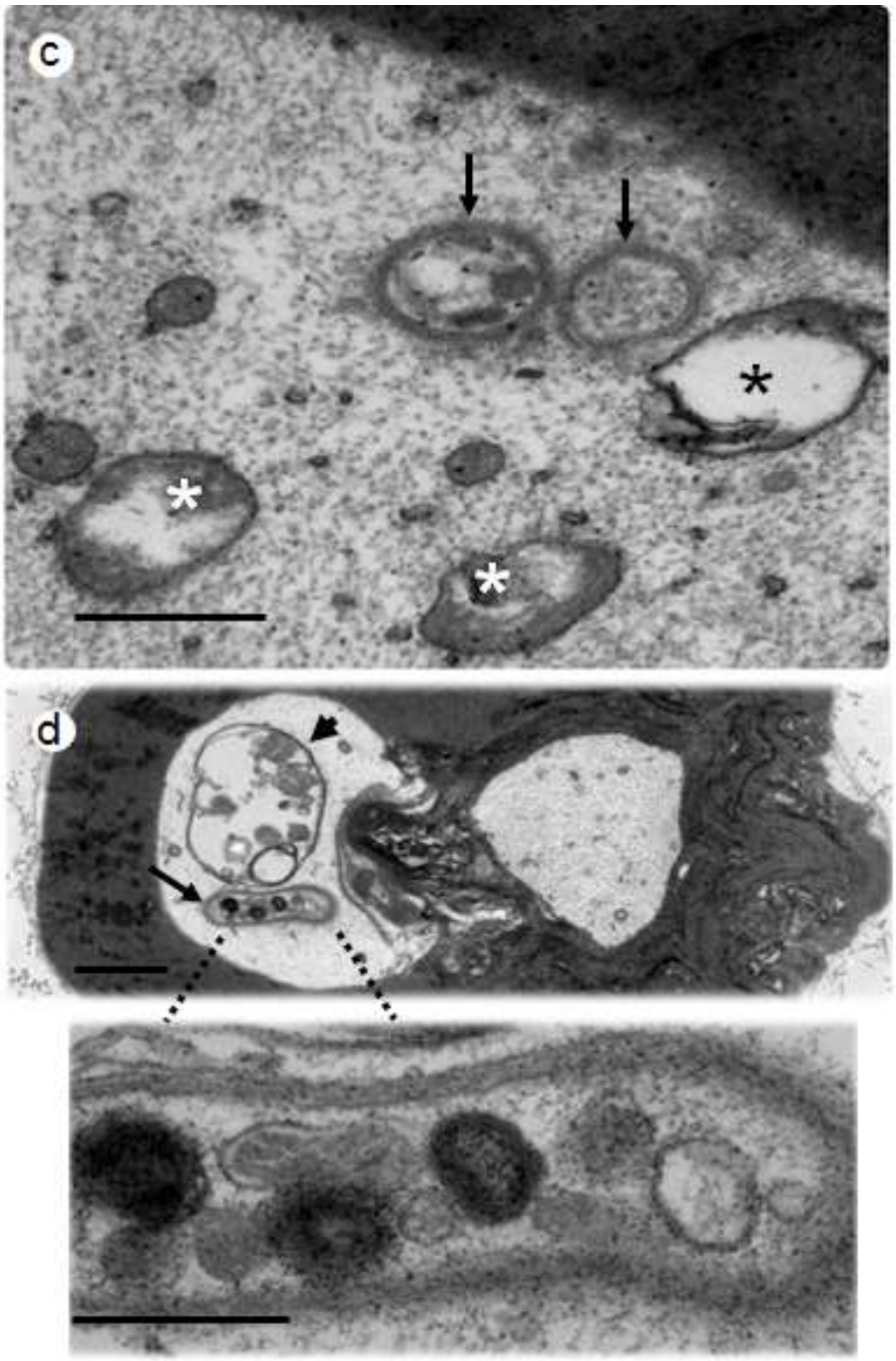

Fig. 3a-d Micrographs showing the various kinds of vacuoles found in axons of hypoglycaemic neurons. Some of the vacuoles were formed by axolemma engulfing axoplasm (a), and others were lucid (b-c; black asterisks) or appeared as enlarged mitochondria with degenerated cristae (c; white asterisk). Also, some of these vacuoles exhibited autophagosome-like structures (i.e. double-membrane vesicles engulfing damaged intracellular structures) engulfing mitochondria and lysosomes (c-d; arrows) or autophagolysosome-like structures (d; arrowhead). Scale bars $=0.5$ $\mu \mathrm{m}$.

At a later stage of degeneration, there was clear breakdown of axons, and myelin sheaths were collapsed and occurred as large clumps in the Schwann cell cytoplasm (Fig. 4a). These neurons contained osmophilic and osmophobic fat droplets, as well as aggresomes (aggregates of damaged proteins) which are revealed by electron 
microscopy as electron-dense inclusion bodies in the cytoplasm. The degradation of myelin occurred not only in Schwann cells, but also in macrophages which were numerous in the LPNs and VRs (Fig. 4b-c). Macrophages were mostly located near or in close contact with denervated Schwann cells (Fig. 4c), and often contained two or more myelin clumps, as well as myelin degradation products, fat droplets, and single- and multilayered vesicles surrounding other organelles (Fig. 4b-d). In one semin-thin section of the LPNs, there were 18 cells involved in degradation of myelin, $61 \%$ of which were Schwann cells and $39 \%$ macrophages (Table 1 ). In one semithin section of the VRs, 50 cells were involved in degradation of myelin, $42 \%$ of which were Schwann cells and 58\% macrophages; and dorsal roots revealed only 11 degenerating myelin, and $73 \%$ of that degradation occurred in Schwann cells (Table 1). In addition, myelin debris that had no connection with Schwann cells or macrophages was observed in the endoneurium of the damaged nerves, suggesting that myelin degenerate more slowly than other parts of the Schwann. Probably these myelin fragments would eventually be discovered by macrophages.

The plantar nerves and VRs also displayed signs of regenerating axons in different stages of development (i.e., from formation of bands of Büngner to well-developed regeneration units). Examination of one semi-thin section from each of six animals showed that there were 47 and 2 regeneration units in the LPNs and VRs, respectively (Table 1). Myelin debris was observed in cells that were enclosed by a basal lamina. Structures associated with autophagy (i.e., phagophores, autophagosomes, lysosomes, and autolysosomes) were frequently observed in the cytoplasm of the Schwann cells in the bands of Bünger (Fig. 5a-c). Moreover, small 
fragments of myelin debris, fat droplets, lysosomes, and autophagosome-like

structures were found in some of the regenerated axons and in the cytoplasm of

Schwann cells associated with those axons (Fig. 5a-c).
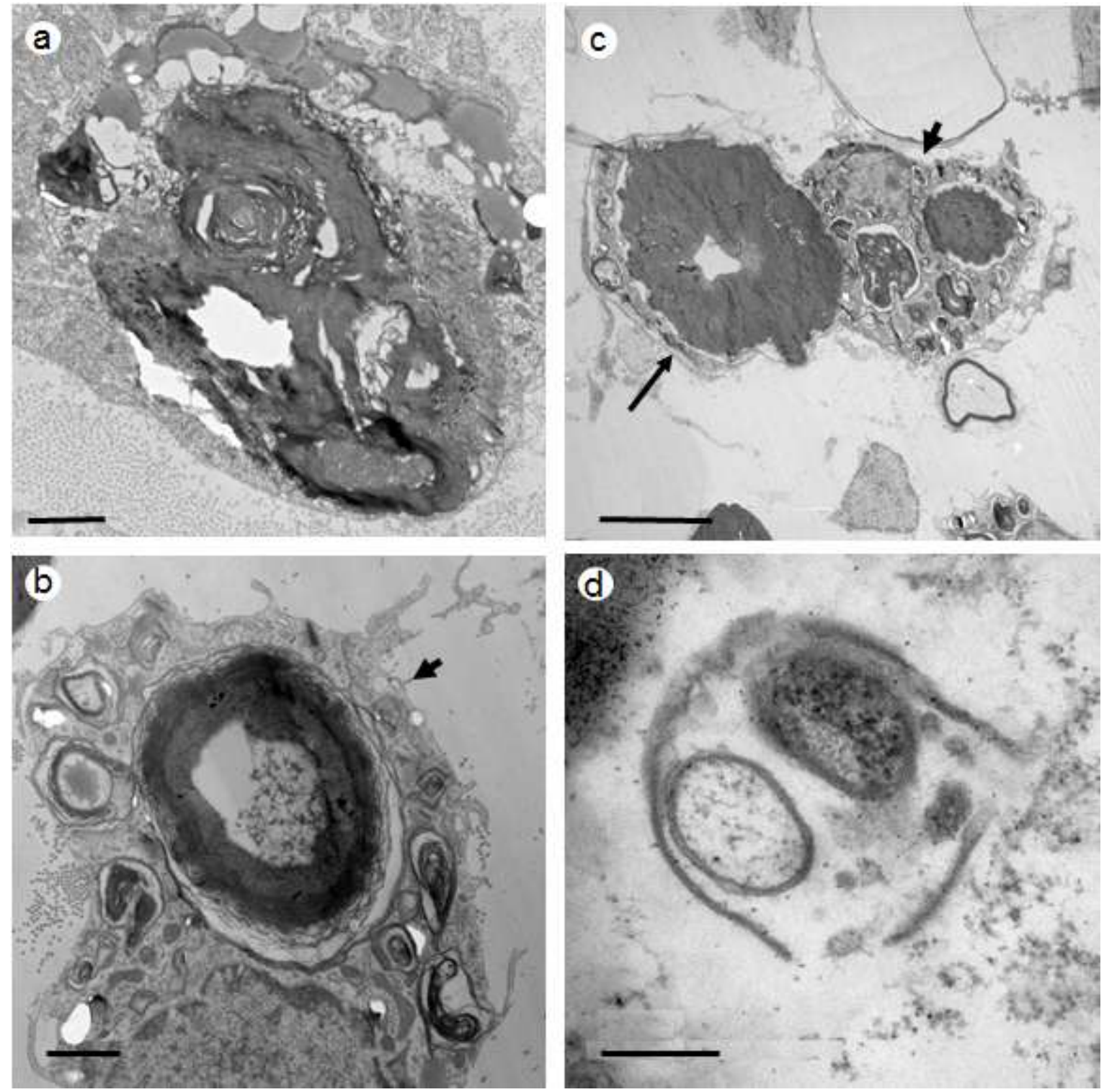

Fig. 4a-d Myelin debris and osmophilic and osmophobic fat droplets in denervated Schwann cells (a) and macrophages (b) in the nerves of insulin-treated diabetic hypoglycaemic rats. (c) Macrophages (arrowhead) often occurred in close contact with a denervated Schwann cell (arrow). Multilayered vesicles (b-c) and heterophagosomes (i.e., cytoplasmic vacuoles including phagocytosed exogenous inclusions) (d) were observed in macrophages. Scale bars: $1 \mu \mathrm{m}$ (a and b); $5 \mu \mathrm{m}$ (c); $0.25 \mu \mathrm{m}(\mathbf{d})$. 

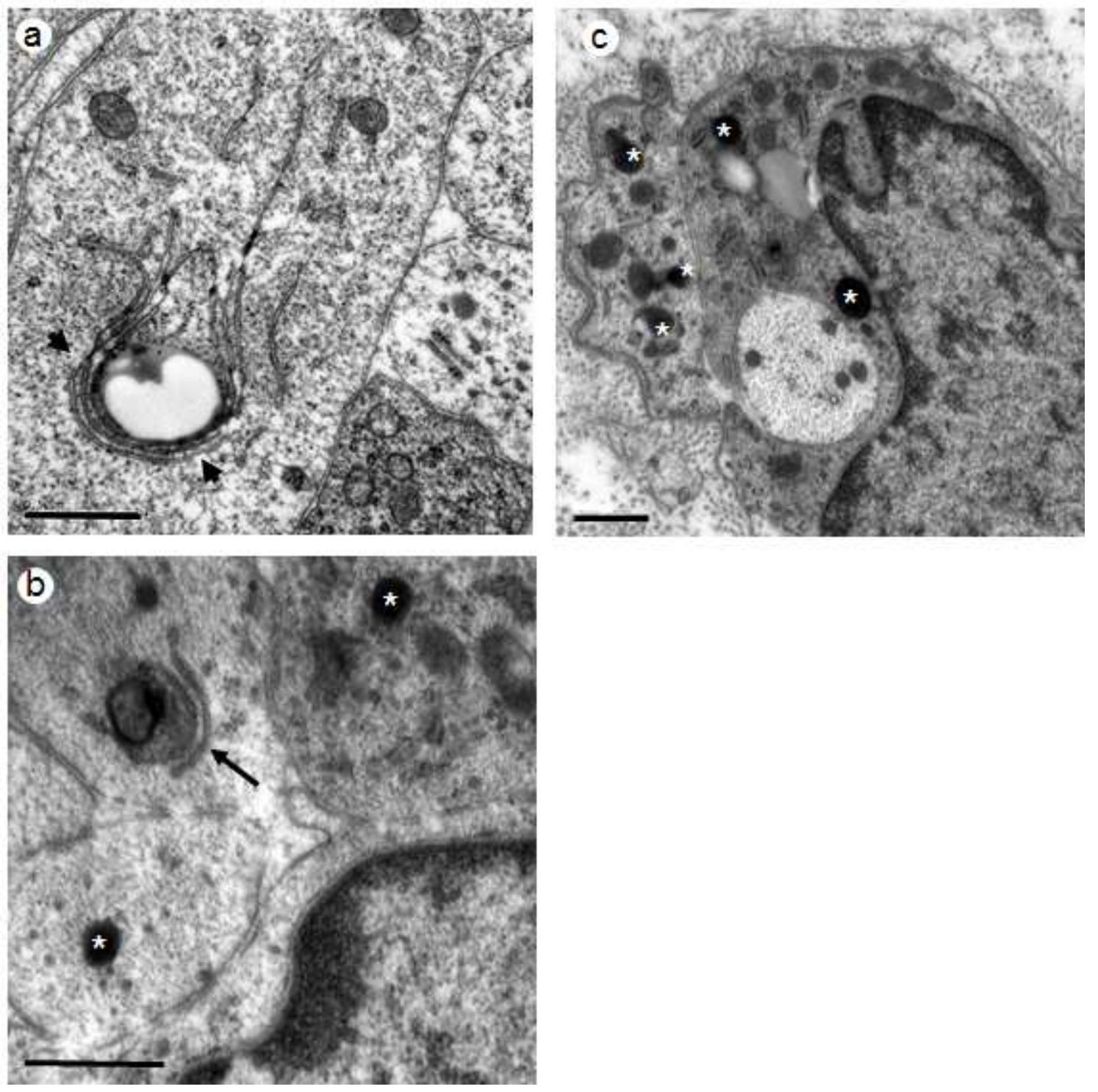

Fig. 5a-c Micrographs showing the following autophagy-associated structures, which were frequently found in the cells of bands of Bünger of insulin-treated diabetic hypoglycaemic nerves: isolated membranes called autophagophores in the regenerated axons (a; arrowheads), lysosomes (b-d; asterisks), and autophagosomes (c; arrow). Scale bars: $0.5 \mu \mathrm{m}$ (a and $\mathbf{c})$ and $0.2 \mu \mathrm{m}$ (b).

Unmyelinated nerve fibres seemed to be normal, except for the occurrence of small vesicle-like lucid structures with diameters ranging from 0.1 to less than $0.3 \mu \mathrm{m}$. 


\section{DISCUSSION}

In the present study, lateral plantar nerves, dorsal roots, and ventral roots from insulin-treated diabetic hypoglycaemic rats were examined for ultrastructural signs of ongoing autophagy. The results revealed the presence of a few autophagy associated vacuoles in myelinated axons exhibiting early pathological changes. At a later stage, when the axons had degenerated, the process of myelin degradation occurred in the denervated Schwann cells without any convincing evidence of autophagy. Extensive signs of heterophagy (i.e., digestion of exogenous particles) were detected in macrophages, many of which had engulfed more than one Schwann cell. There were also indications of ongoing autophagy during axonal regeneration; these consisted mainly of autophagophores (i.e., isolated membranes in the cytoplasm that eventually form autophagosomes) and lysosomes in cells in bands of Büngner, and a large proportion of the autophagophores contained fat droplets or myelin debris. These results indicate the following: (a) peripheral axons have access to the biological machinery of autophagy; (b) Schwann cells that lose axonal connection do not survive; (c) macrophages play a crucial role in clearance of damaged nerve fibres; (d) autophagy occurs in both axons and Schwann cells during axonal regeneration.

Diabetic BB/Wor rats were used as an animal model in the present study. As mentioned, some rats of this strain develop diabetes spontaneously after autoimmune inflammation in the pancreas. ${ }^{24}$ Here, hypoglycaemia was achieved using subcutaneous insulin implants that release two units of bovine insulin per day over a period of four to six weeks. There were a number of reasons for choosing to use diabetic BB/Wor rats. First, because it is easier to achieve a relatively stable 
insulin-induced hypoglycaemic regimen in such animals, because the diabetic body lacks the ability to regulate the level of glucose in the blood as a part of metabolic homeostasis. Second, according to earlier results that our team obtained using diabetic BB/Wor rats, the insulin implants are convenient to use and are also better from the animals' perspective, considering that they are only $7 \mathrm{~mm}$ long and $2 \mathrm{~mm}$ in diameter, and can be easily inserted subcutaneously in the neck once every four weeks. An alternative animal model would have been to administer insulin to healthy rats. However, maintaining a similar hypoglycaemic status in healthy animals probably would have required occasional insulin injections in addition to insulin implants to counteract the natural response of body physiology to preserve a normal blood glucose level, and administering injections over a long period of time would have been stressful for the animals. Third, previous studies by our group ${ }^{19-23}$ had shown that hypoglycaemia, but not chronic hyperglycaemia, in diabetic BB/Wor rats leads to development of peripheral neuropathy combined with axonal de-and regeneration. Hence, it seemed appropriate to use this animal model to examine autophagy in association with peripheral neuropathy caused by hypoglycaemia.

The current results revealed enlarged disrupted mitochondria in the axons of hypoglycaemic nerves. The most important function of mitochondria is to generate ATP, which is highly critical for maintaining neuronal homeostasis, and thus the occurrence of abnormal mitochondria in the investigated axons indicates that it is difficult for hypoglycaemic nerves to cope with insufficient local ATP production. When the access to energy is suboptimal, autophagy may compensate for the loss of mitochondrial function by recycling damaged cellular contents. However, chronic hypoglycaemia eventually causes degeneration of large myelinated axons, ${ }^{19-23}$ which 
suggests that this compensatory strategy, if it actually exists, is not the most advantageous for large fibres during prolonged energy deprivation.

Denervated Schwann cells in the current samples also contained aggresomes, which are membrane-free structures that consist of accumulations of misfolded proteins ${ }^{25}$ and are formed when proteosomal activity is inhibited. ${ }^{26}$ Schwann cells eliminate aggresomes by a mechanism that is enhanced when autophagy is activated. ${ }^{27}$ Although the denervated Schwann cells studied here did harbour aggresomes, they showed no obvious signs of autophagy. It is possible that these aggresomes, which were composed mostly of myelin-associated proteins, were formed due to saturation of degradation pathways. Such accumulation of proteins per se may be toxic to the cells and contribute to development of peripheral neuropathies. ${ }^{28}$ Involvement of macrophages in phagocytosis of denervated Schwann cells indicates that, even if the process of autophagy-lysosomal degradation does occur in Schwann cells, it alone will not be able to manage degradation of the substantial amount of damaged organelles, but will require the assistance of macrophages to complete that task.

As mentioned above, accumulation of autophagic vacuoles in neurons has been observed in several neurodegenerative disorders in the CNS. In the PNS, autophagy vacuoles have been detected in axons in transected sciatic nerves ${ }^{18}$ and in sympathetic neurites subjected to harmful stimulation in vitro. ${ }^{17}$ It is not known whether the accumulation of autophagy-related structures is due to induction of autophagy or to disturbed clearance of autophagic vacuoles. Some investigators contend that autophagy protects neurons from degeneration, and that dysregulation of autophagy and impaired autophagic clearance may promote development of 
neurodegenerative diseases..$^{29-32}$ Other researchers suggest that autophagy is a mechanism of cell death rather than cell survival. ${ }^{33,34}$ Nevertheless, the Nomenclature Committee on Cell Death ${ }^{35}$ has classified autophagy as a cell death mechanism based on its morphological appearance. In the current study, autophagyrelated structures were observed in the large myelinated axons, but it is not clear whether these axons would eventually have died or if they could have survived the insult. What is more apparent is that death of denervated Schwann cells was induced by activation of heterophagy in macrophages, or, in other words, by the Schwann cells being phagocytosed and digested in vacuoles that fused with lysosomes in the macrophages.

In Wallerian degeneration, injured axons sprout new branches from a healthy node proximal to the site of injury, and these branches grow inside the basal lamina that belonged to their parent axons. Schwann cells compete with each other to ensheath and myelinate the new axonal branches, and those that are successful in this competition survive. The present results indicated extensive signs of autophagy in regenerated axons and their associated Schwann cells, as well as in the Schwann cells that lacked axonal connection. This demonstrates that Schwann cells have the biological machinery that is necessary for autophagy, and this system is activated during axonal regeneration rather than degeneration. Why would Schwann cells initiate a cell survival mechanism when they have lost their main goal for survival which is to support their associated axons? During regeneration, however, Schwann cells struggle to survive, because they are needed by the newly forming axons. 
In conclusion, the results of the current study suggest that autophagy is involved in degeneration and regeneration of hypoglycaemic axons and the role of this process is probably more closely related to cell survival than to cell death. Obviously, this matter remains to be clarified. It is plausible that autophagy plays disparate roles in different cells, depending on the cell type, the tissue involved, and the cause of damage, as well as the prerequisite for a successful outcome of cell repair. It is imperative to use biochemical methods to further investigate the involvement of autophagy in peripheral axons, Schwann cells, and macrophages in order to better understand the role of such cellular mechanism in peripheral nerve damage and repair.

\section{Acknowledgements}

This study was supported by Östergötland County and by the Faculty of Health Sciences of Linköping University. I express my sincere gratitude to Dr. Chonghe Jiang for preparing the illustrations used in this paper.

\section{Conflict of interest}

The author declares no conflict of interest. 


\section{References}

1. Waller AV. Experiments on the section of the glossopharyngeal and hypoglossal nerves of the frog, and observations of the alterations produced thereby in the structure of their primitive fibres. Philos Trans R Soc. Lond. B Biol Sci 1850; 140: 423-9.

2. Mattson MP, Keller JN, Begley JG. Evidence for synaptic apoptosis. Exp Neurol 1998; 153: 35-48.

3. Finn JT, Weil M, Archer F, et al. Evidence that Wallerian degeneration and localized axon degeneration induced by local neurotrophin deprivation do not involve caspases. J Neurosci 2000; 20: 1333-41.

4. Grinspan JB, Marchionni MA, Reeves M, et al. Axonal interactions regulate Schwann cell apoptosis in developing peripheral nerve: neuregulin receptors and the role of neuregulins. J Neurosci 1996; 16: 6107-18.

5. Marchionni MA, Kirk CJ, Isaacs IJ. Neuregulins as potential drugs for neurological disorders. Cold Spring Harb Symp Quant Biol 1996; 61:459-72.

6. Syroid DE, Maycox PR, Burrola PG. Cell death in the Schwann cell lineage and its regulation by neuregulin. Proc Natl Acad Sci U S A 1996; 93: 9229-34.

7. Bruck, W. The role of macrophages in Wallerian degeneration. Brain Pathol 1997; 7: 741-752.

8. Mueller M, Wacker K, Ringelstein EB. Rapid response of identified resident endoneurial macrophages to nerve injury. Am J Pathol 2001; 159: 2187-97. 
9. Hughes PM, Wells GM, Perry VH, et al. Comparison of matrix metalloproteinase expression during Wallerian degeneration in the central and peripheral nervous systems. Neuroscience 2002; 113: 273-87.

10. Shacka JJ, Roth KA, Zhang J. The autophagy-lysosomal degradation pathway: role in neurodegenerative disease and therapy. Front Biosci 2008; 13: 718-36.

11. Nixon RA, Wegiel J, Kumar A. Extensive involvement of autophagy in Alzheimer disease: an immuno-electron microscopy study. J Neuropathol Exp Neurol 2005; 64: 113-122.

12. Anglade $\mathrm{P}$, Vyas $\mathrm{S}$, Javoy-Agid $\mathrm{F}$, et al. Apoptosis and autophagy in nigral neurons of patients with Parkinson's disease. Histol Histopathol 1997; 12: 25-31.

13. Hara T, Nakamura K, Matsui M. et al. Suppression of basal autophagy in neural cells causes neurodegenerative disease in mice. Nature 2006; 441: 885-89.

14. Komatsu M, Waguri S, Chiba T. Loss of autophagy in the central nervous system causes neurodegeneration in mice. Nature 2006; 441: 880-84.

15. Koike M, Shibata M, Tadakoshi M. Inhibition of autophagy prevents hippocampal pyramidal neuron death after hypoxic-ischemic injury. Am J Pathol 2008; 172: 454-69.

16. Uchiyama Y, Koike M, Shibata M. Autophagic neuron death. Methods Enzymol 2009; 453: 33-51.

17. Yang $\mathrm{Y}$, Fukui K, Koike T. Induction of autophagy in neurite degeneration of mouse superior cervical ganglion neurons. Eur J Neurosci 2007; 26: 2979-88.

18. Piao ZX, Wang WS, Xu XJ. Autophagy of neuron axon during regeneration of rat sciatic nerves. Di Yi Jun Yi Da Xue Xue Bao 2004; 24: 361-64. 
19. Jamali R, Mohseni S. Hypoglycaemia causes degeneration of large myelinated nerve fibres in the vagus nerve of insulin-treated diabetic BB/Wor rats. Acta Neuropathol 2005; 109: 198-206.

20. Jamali R, Mohseni S. Differential neuropathies in hyperglycemic and hypoglycemic diabetic rats. J Neuropathol Exp Neurol 2006; 65: 1118-25.

21. Mohseni S. Hypoglycaemic neuropathy in diabetic BB/Wor rats treated with insulin implants affects ventral root axons but not dorsal root axons. Acta Neuropathol 2000; 100: 415-20.

22. Mohseni S, Hildebrand C. Hypoglycaemic neuropathy in BB/Wor rats treated with insulin implants: electron microscopic observations. Acta Neuropathol 1998a; 96 :151-56.

23. Mohseni S, Hildebrand C. Neuropathy in diabetic BB/Wor rats treated with insulin implants. Acta Neuropathol 1998b; 96: 144-50.

24. Marliss EB, Nakhooda AF, Poussier P. The diabetic syndrome of the 'BB' Wistar rat: possible relevance to type 1 (insulin-dependent) diabetes in man. Diabetologia 1982; 22: 225-32.

25. Notterpek L, Ryan MC, Tobler AR. PMP22 accumulation in aggresomes: implications for CMT1A pathology. Neurobiol Dis 1999; 6: 450-60.

26. Johnston JA, Ward CL, Kopito RR. Aggresomes: a cellular response to misfolded proteins. J Cell Biol 1998; 143: 1883-98.

27. Fortun J, Dunn WA Jr, Joy S, et al. Emerging role for autophagy in the removal of aggresomes in Schwann cells. J Neurosci 2003; 23: 10672-80.

28. Kopito RR. Aggresomes, inclusion bodies and protein aggregation. Trends Cell Biol 2000; 10: 524-30. 
29. Alirezaei M, Kiosses WB, Fox HS. Decreased neuronal autophagy in HIV dementia: a mechanism of indirect neurotoxicity. Autophagy 2000; 4: 963-66.

30. Boland B, Kumar A, Lee S, et al. Autophagy induction and autophagosome clearance in neurons: relationship to autophagic pathology in Alzheimer's disease. J Neurosci 2008; 28: 6926-37.

31. Boya P, Gonzalez-Polo RA, Casares N, et al. Inhibition of macroautophagy triggers apoptosis. Mol Cell Biol 2005; 25: 1025-40.

32. Martinez-Vicente M, Cuervo AM. Autophagy and neurodegeneration: when the cleaning crew goes on strike. Lancet Neurol 2007; 6: 352-61.

33. Loos B, Engelbrecht AM. Cell death: a dynamic response concept. Autophagy 2009; 5: 590-603.

34. Yue Z, Friedman L, Komatsu M. The Cellular Pathways of Neuronal Autophagy and Their Implication in Neurodegenerative Diseases. Biochim Biophys Acta 2009; 1793: 1496-507.

35. Kroemer G, Galluzzi L, Vandenabeele P. Classification of cell death: recommendations of the Nomenclature Committee on Cell Death 2009. Cell Death Differ 2009; 16: 3-11. 

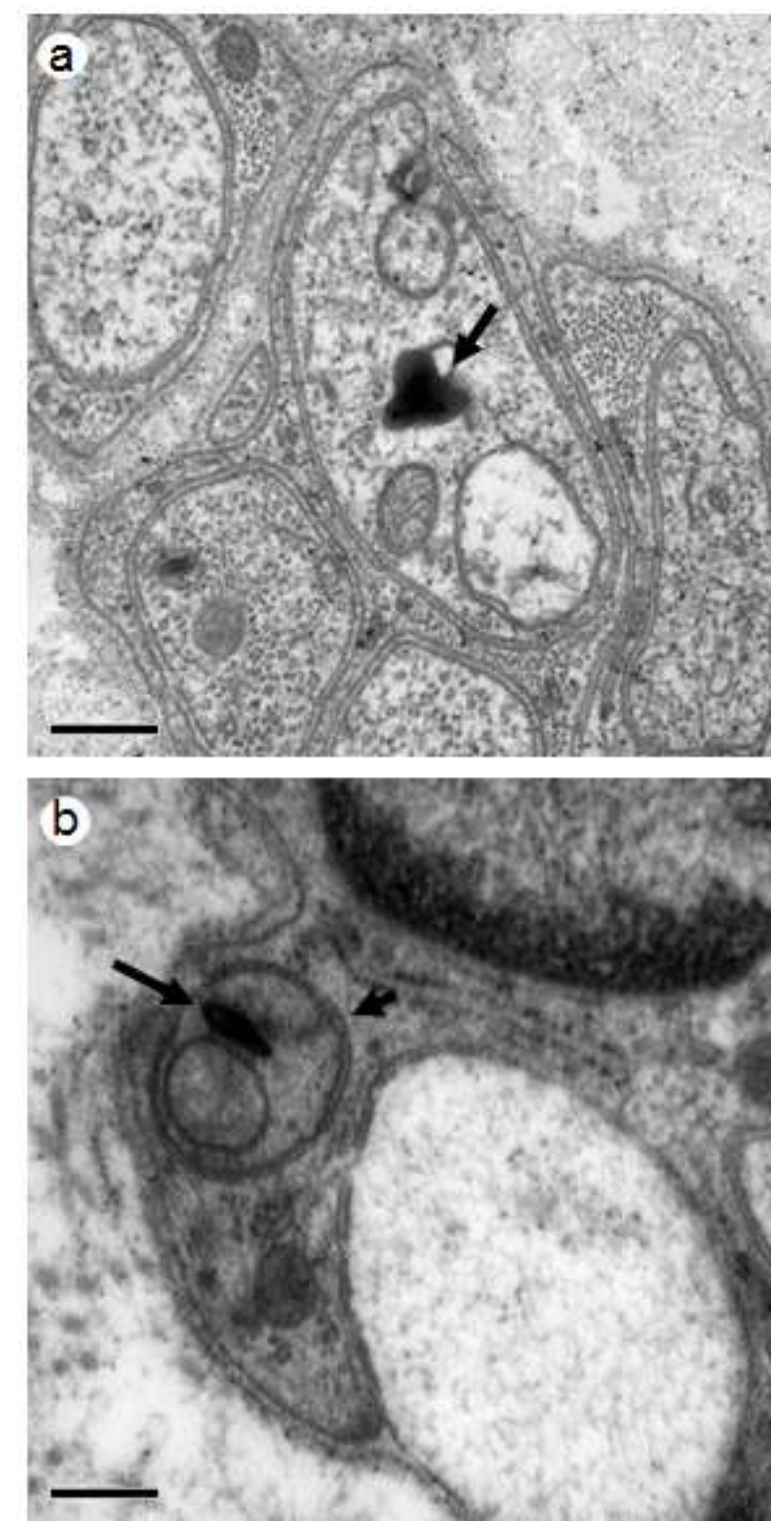

Fig. 6a-b Regenerated axons and their associated Schwann cells in hypoglycaemic nerves contained small fragments of myelin debris (arrows), electron lucid vacuoles, and autophagosomes (arrowhead). Scale bars: $0.5 \mu \mathrm{m}$ (a) and $0.2 \mu \mathrm{m}$ (b). 